\title{
Assessment of Surgical Correction at Vascular Ring Anomalies
}

\author{
Çağatay Bilen ${ }^{1}$, Gökmen Akkaya ${ }^{1}$, Osman Nuri Tuncer ${ }^{1}$, Mehmet Fatih Ayık ${ }^{1}$, Yüksel Atay ${ }^{1}$
}

1) Ege University School of Medicine, Department of Cardiovascular Surgery, Izmir, Turkey

\begin{abstract}
Aim: In this retrospective study, we present our fifteen years of experience in surgical treatment in vascular rings. Thus, we aimed to determine the factors that causing improved both early and late term complications.

Methods: Sixteen patients who underwent surgical treatment with the diagnosis of vascular ring between 2003-2018 were included in this study. Hospital records were analyzed retrospectively. Demographic data of the patients, preoperative symptoms and postoperative outcomes were evaluated.

Results: The patients were mean $43.50 \pm 38.61$ months of age and weighed $26.00 \pm 13.81 \mathrm{~kg}$ at the time of surgeries. 6 patients (37.5\%) were diagnosed with double aortic arch, 7 patients (43.7\%) had left arcus aorta associated with right aberrant subclavian artery and 3 patients $(18.7 \%)$ had right arcus aorta and left ligamentum arteriosum. The most common symptom was cough followed by shortness of breath, difficulty in swallowing, reflux, recurrent pneumonia respectively. One patient had atrial septal defect, another patient had ductus arteriosus and another patient had ventricular septal defect as a coexisting cardiac disorder. As early complications, we observed cylothorax, pneumothorax and need of tracheostomy due to failure of extubation in three cases. Mean follow up time was $7.00 \pm 3.57$ years. Patients had no complaints except for one case with asthma. However, there was no pressure measured on trachea in that patient. No patients died during hospitalizations or follow-ups.

Conclusion: Vascular rings can manifest with various symptoms at different ages and many patients also have co-existing cardiac anomalies. However, once the diagnosis is confirmed, surgical approach is essential and leads to satisfactory, uneventful long-term survival results.
\end{abstract}

Keywords: Vascular ring, double aortic arc, aberrant right subclavian artery 


\section{Introduction}

Vascular rings involve an abnormal development of aortic arch and branches during embryological development in neonatal period which causes compression on trachea and/or esophagus; resulting in symptoms such as cough, recurrent respiratory infections, wheezing, dysphagia or respiratory distress. Most frequent ones are wheezing and stridor respectively. ${ }^{(1,2)}$ Forms of the diseases are mainly divided into two groups according to total or partial encirclement of the trachea and esophagus. The most common vascular rings forms are double aortic arch and right aortic arch with left ligamentum. ${ }^{(1,3)}$ Vascular rings constitute less than $1 \%$ of among congenital cardiac disorder. ${ }^{(4)}$

However, the recurrent and progressive clinical findings associated with vascular rings, depending on the severity of the trachea-esophageal pressure, may require urgent intervention, and they need to be profoundly examined. The diagnosis can be confirmed using various imaging and endoscopic methods such as computer assisted tomography (CT), magnetic resonance imaging (MRI), bronchoscopy, gastroscopy, echocardiography, cardiac catheterization, and upper GI series. ${ }^{(5)}$ Once the patient was diagnosed, surgical treatment is required for decompression as a standard therapy. Despite surgical techniques often differ depending on the anatomical features of the disease, several reports indicate satisfactory results with low mortality and event-free long-term outcomes. Nevertheless, the respiratory problem may continue after the operation due to structural defect and acquired tracheomalacia secondary to long-lasting pressure on the airways. ${ }^{(6,7)}$

Herein, we present our ten years of early and late results of the patients who were operated at our hospital with the diagnosis of vascular ring. Thus, we aimed to determine the significant points of clinical pattern at presentation, optimal time and efficiency of surgical operations.

\section{Material and Methods}

The patients who were operated with the diagnosis of vascular ring between 2005-2017 were included in this study. Hospital records were retrospectively analyzed. Demographic data of the patients, preoperative symptoms such as cough, dyspnea, dysphagia, wheezing, stridor or repetitive respiratory infections and postoperative outcomes were evaluated.

The imaging methods which applied aiming to confirm the diagnosis were presented below. Those are consisted on transthoracic echocardiography (TTE), computed thorax angiography (CTA), magnetic resonance angiography (MRA), bronchoscopy and barium esophagography.

For classification of the patients, the classification method which was described and modified afterwards by Congenital Heart Surgery Nomenclature and Database Project was utilized. ${ }^{(8)}$ According to this, vascular rings were divided into six main subgroups which are defined as double aortic arch, right arcus aorta + left ligamentum arteriosus, compression of the innominate artery, pulmonary artery sling, left arcus aorta + right aberran subclavian artery, respectively.

Operative technique, duration in intensive care unit (ICU), extubation time, duration in hospital and postoperative complications were recorded.

\section{Operative Techniques}

In double aortic arch patients, following posterolateral thoracotomy incision, plevral cavity was accessed through fourth intercostal space. Both aortic arches and ligamentum arteriosum was released and ligamentum arteriosum was divided. Initially, diameters of the arches were evaluated. By dividing the hypoplasic one, trachea and esophagus were decompressed. In the cases where both diameters were similar, blood pressure of limbs were measured, thereafter, the arch which was located at the same side with the lower arterial pressure was divided. Furthermore, right arch divison was chosen due to circumscribing trachea, in presence of equality in both diameter size and blood pressure measurement. However, descending part of the divided arch was stabilized at thorax wall via polyprolene stitches, thereby recompression was avoided. Mediastinal pleura was always kept open. ${ }^{(9,10)}$

In cases with right arcus aorta and left ligamentum arteriosum, after posterolateral thoracotomy incision, thoracal cavity was accessed through fourth intercostal space and ligamentum arteriosum was cut away. Also, in patients with Kommerell diverticulum, the diverticulum was excised. 
Left thoracotomy was performed for surgical approach in patients with aberrant right subclavian artery. Then, aberrant vessel was divided and anastomosed with right carotid artery.

\section{Statistical Analysis}

Descriptive data were expressed in mean, standard deviation, median values. The data were recorded on a computerized database and analyzed using SPSS version 20.0 statistical software (SPSS Inc., Chicago, Illinois, USA).

\section{Results}

Overall 16 patients were operated for vascular ring anomalies. The patients had mean $43.50 \pm 38.61$ months of age. Mean weight of the patients was measured as $26.00 \pm 13.81 \mathrm{~kg}$ preoperatively. Seven out of sixteen patients were female, while nine were male.

There was double aortic arch in six patients, left arcus aorta associated with right aberrant subclavian artery in seven patients, right arcus aorta and left ligamentum arteriosum in three patients. Moreover, one patient had Kommerell diverticle. In addition to these pathologies, associated diseases were diagnosed in four patients; these were atrial septal defect (ASD), ventricular septal defect (VSD), patent ductus arteriosus (PDA) and diaphragmatic hernia. Demographic data and clinic features were shown in Table 1.

Most common symptom was coughing among patients. Dyspnea and dysphagia were following respectively. Besides that, acid reflux, cyanosis and recurrent respiratory infections were less frequent. While respiratory symptoms were mostly seen in patients with double aortic arch and right arcus aorta and ligamentum arteriosum, dysphagia was much more common in patients with aberrant right subclavian artery.

All paitents were examined via TTE. The definitive diagnosis was made by MRA in three patients and CTA in 13 patients. Due to the tracheomalacia, broncoscopy and barium esophagography were applied to one patient each. Imaging methods were presented in Table 2.

Mean entubation time and duration in intensive care unit were $9.31 \pm 16.79,32.62 \pm 26.33$ hours, respectively. Hospitalization time was calculated as $6.31 \pm 2.62$ days. ASD and VSD closure were performed under cardio-

Table 1. Demographic data and clinic features

Caracteristics

Sex (Male/Female)

Age (Months)

Wight (kg)

Symptoms \%

Cough

43.75

Shortness of breath

Difficulty in swallowing

Recurrent pneumonia

Reflux

\section{Echocardiographic findings}

ASD 1

VSD 1

PDA 1

Dignosis

Double arch 6

Right arch + right ligamentum 3

Arteriosum

Aberrant right subclavian artery 7

VSD: vetricular septal defect; ASD: atrial septal defekt; $P D A$ patent ductus arteriosus

Data are presented as mean $\pm S D$ or number

pulmonary by-pass, however PDA division was utilized concurrently. Early complications were observed in 3 patients. These were pneumothorax, cheilothorax and tracheostomy application due to failure of extubation in one patient who had tracheomalacia preoperatively. Postoperative mean follow-up time was $7.00 \pm 3.57$ years. Postoperative complications and intensive care parameters were indicated in Table 3.

\section{Discussion}

Various vascular ring formations requires different surgical approaches; thus, treatment is still challenging. Furthermore, vascular rings manifestation varies from severe respiratory problems occur in postnatal period to 
Table 2. Diagnostic tests for vascular ring

\begin{tabular}{|c|c|c|c|c|c|c|}
\hline $\begin{array}{l}\text { Patient } \\
\text { ID no. }\end{array}$ & $\begin{array}{l}\text { Bronchog } \\
\text { copy }\end{array}$ & Echocardio raphy & $\begin{array}{c}\text { Barium swallow } \\
\text { X-ray }\end{array}$ & CT ang ography & MRI & Bronchoscopy \\
\hline 1 & & $V S D$ & - & + & - & - \\
\hline 2 & - & $N / A$ & + & + & - & - \\
\hline 3 & - & $N / A$ & - & + & - & - \\
\hline 4 & - & $N / A$ & - & + & - & - \\
\hline 5 & - & $N / A$ & - & + & - & - \\
\hline 6 & - & $N / A$ & - & - & + & - \\
\hline 7 & - & $N / A$ & - & + & - & - \\
\hline 8 & + & $N / A$ & - & + & - & - \\
\hline 9 & - & $N / A$ & - & + & - & + \\
\hline 10 & - & $A S D$ & - & - & + & - \\
\hline 11 & - & $P D A$ & - & + & - & - \\
\hline 12 & - & $N / A$ & - & + & - & - \\
\hline 13 & - & $N / A$ & - & - & + & - \\
\hline 14 & - & $N / A$ & - & + & - & - \\
\hline 15 & - & $N / A$ & - & + & - & - \\
\hline 16 & - & $N / A$ & - & + & - & - \\
\hline
\end{tabular}

Table 3. Postoperative complications and intensive care parameters

\begin{tabular}{|l|c|}
\hline & $\mathbf{n}$ \\
\hline Duration of ventilation (hours) & $9.31 \pm 16.79$ \\
\hline Duration in intensive care unit (hours) & $32.62 \pm 26.33$ \\
\hline Hospital stay (days) & $6.31 \pm 2.62$ \\
\hline Complications & 1 \\
\hline Pneumothorax & 1 \\
\hline Pneumothorax & 1 \\
\hline Extubation failure & \\
\hline
\end{tabular}

nonspecific findings in school age depending on the degree of the compression over trachea and/or esophagus.
In such cases, the diagnosis may be overlooked and incidentally noticed while examining less typical symptoms. As an example, exercise induced dyspnea and asthma was observed in our patients. Nonetheless, when the literature was reviewed, similarly to us, Sturm and colleagues ${ }^{(11)}$ reported an 8 years old case of who was being treated with the diagnosis of asthma over five years. Patients presented in a wide range of age between 4-132 months at the time of diagnosis with a multitude of signs and symptoms; cough was the most common symptom, followed by shortness of breath, difficulty in swallowing, recurrent pneumonia and acid reflux, respectively. In presence of these repetitive sings, pediatrician should be alerted about vascular ring.

Currently, non-invasive methods such as TTE, MRA and BTA replaced previously widely used diagnosis techniques. However, in three of our patients, broncoscopy, 
Figure 1. Computed tomographic angiography image of a double aortic arch. Hypoplastic aortic arch marked in red circle, compressed esophagus circled in yellow.

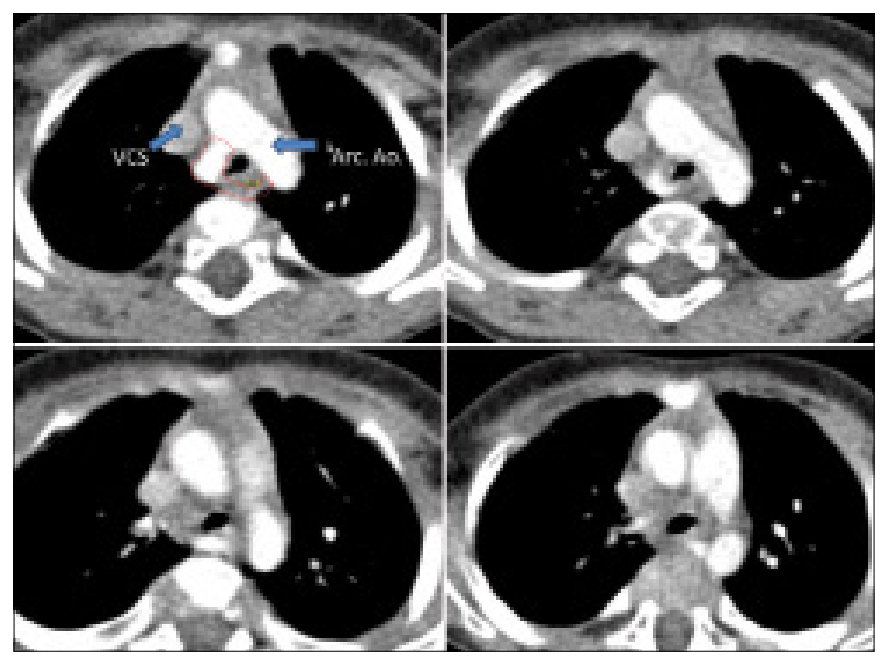

Figure 2. Computed tomographic angiography image an aberrant right subclavian artery. Right aberrant subclavian artery circled in red.

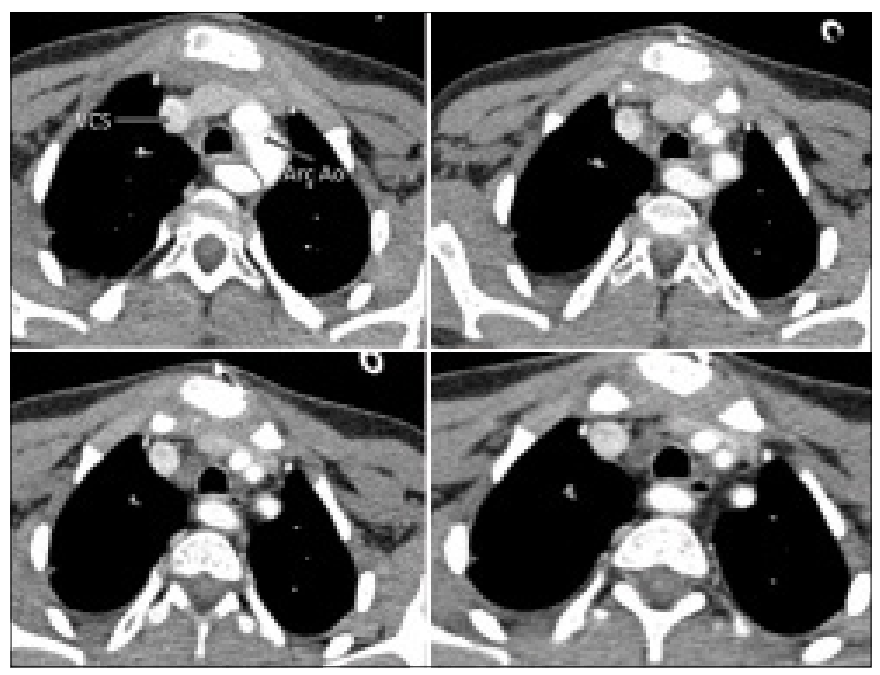

eosophagoscopy and barium swallow x-ray are required for diagnosis. Regardless of how the patient was diagnosed, all patients should be initially examined via TTE for concurrent cardiac anomalies. Sometimes TTE may be incapable and further, more detailed methods may be needed. While cardiac catheterization and angiography were mostly utilized in the past, nowadays, they were abandoned in favor of CTA and MRA. We detected three accompanying congenital cardiac diseases; and encountered PDA, VSD and ASD in one patient each. Overall incidence of concomitant cardiac anomalies has been stated between $12-19 \%$ in recent studies compatible with our outcomes. ${ }^{(4)}$
Despite we prefer posterolateral thoracotomy in most cases, in case of cardiopulmonary by-pass requirement owing to intracardiac pathologies, median sternal access is necessary.

Vascular rings are classified under six main subgroups, which are: double aortic arch occurring due to the persistence of the fourth right aortic arch, right-sided aortic arch + aberrant left subclavian artery developing as a result of persistence of the aortic arch and regression of the fourth left arch between left subclavian artery and the left carotid artery and left aortic arch + aberrant right subclavian artery (ARSA) occurs folowing regression of the fourth right arch between the carotid and subclavian arteries. Our series include only three of these.

This classification is revised by Becker et al. ${ }^{(1)}$ in 1999 and guided our study. In our series, the most frequent form was aberrant right subclavian artery. Recent articles indicate that the majority of cases with left aortic arch and ARSA stay asymptomatic, whereas some exhibited dysphagia or vomiting owing to esophageal compression. ${ }^{(12)}$ However, contrary to this, respiratory symptoms dominancy in such cases has also been reported. ${ }^{(13)}$ Possible underlying mechanism is occurrence of complete circumscription in presence of coexisting right ligamentum arteriosum. ${ }^{(14)}$

In surgical treatment, besides, some authors declare that only the excision of the ARSA provides sufficient treatment; afterwards, although rare, limb ischemia may develop. Aiming to avoid this, mostly anastomosis of ARSA to left subclavian artery performed by various authors. Kinoğlu et al. ${ }^{(15)}$ reported two cases that limb blood flow was ensured via Gore-Tex graft interposition in one case and end-to-side anastomosis in other. Diversely, we have performed ARSA anastomosis with right carotid artery in three cases so far and no ischemia occurred in those. Therefore, we state that this method is feasible and reliable.

Second most frequent type of vascular ring was double aortic arch in our serial. Before surgical repair a detailed investigation is important to determine which arch is dominant. Surgical approach is recommended to be performed through opposite side of the dominant arch. 
We prefer posterolateral thoracotomy using a muscle sparing technique. After entering thoracal space through the fourth intercostal space, followed by lung retraction and opening overlying pleura on the vascular ring, a careful dissection is essential to identify ligamentum arteriosum, the subclavian artery and distal arch.

During this process, surgeons should identify and pay maximal attention to avoid injuring the phrenic, vagal and recurrent laryngeal nerve and the thoracic duct, particularly in the left chest. However, in cases of balanced diameter size in both arches, blood pressure measurement is required to choose which one will be dissected. Another landmark is to leave open the mediastinal pleura at the end of the surgery. Backer et al. ${ }^{(16)}$ state that hereby, surgeons may lead to recurrent scar tissue formation and cause of recurrent stenosis in the area of ring division. In other respects, a compression on the trachea and esophagus caused by any bleeding points or fluid can be avoided.

Other three patients of us had right arch + right ligamentum arteriosum. One of these casea had a Kommerell diverticulum which is a remnant of the posterior left fourth aortic arch in patients with right aortic arch and aberrant left subclavian artery. In addition to the usual surgical method, removal of Kommerell diverticulum is suggested by numerous authors, if left untreated, patients should be kept in close follow-up to detect early aneurysm formation. Evidence of cyctic medial necrosis in resected segments strengthen aforementioned hypothesis. Luciano et al ${ }^{(17)}$ also suggest resection of Kommerell diverticulum in the less frequent cases such as vascular ring with a right aortic arch, a left brachiocephalic artery while a posterior diverticulum attached to a left ductal remnant.

Some centers prefer video-assisted thoracoscopic surgery (VATS) division of vascular rings. ${ }^{(18,19)}$ Riggle et al. ${ }^{(18)}$ reported that, VAT is a feasible method that can be performed with satisfactory results. They have reduced operative time, decreased ICU admissions, chest tube use, chylothorax and overall short length of stay. Nonetheless, despite they have no experience on Vat surgery, Backer at al. ${ }^{(16)}$ underlying a potential risk of massive bleeding that may not be possible to control by a clip or staple line slip off a vessel, especially the way the arches locate behind the esophagus. Moreover, they suggest that it should be keep in mind that atretic arch actually has a small patent lumen.

Beyond bleeding, there are several complications for vascular ring surgery described so far, such as vocal cord paralysis, pneumonia, pneumothorax, chylothorax, Horner's syndrome, neuralgia. ${ }^{(20,21)}$ We had experienced one case each with chylothorax, pneumothorax and tracheostomy application owing to extubation failure who had diagnosed with tracheomalacia preoperatively. In the literature, complications rates have a range of $(10-27 \%),{ }^{(4,6,16-19)}$. However, reported mortality rates are below $1 \%{ }^{(4)}$ We also had no mortality in our 16 patients during hospital stay or follow up. Naimo et al. presented their 36 years of experience in vascular rings and observed no hospital mortality except for 2 cases with Noonan syndrome. Moreover, they indicate that freedom from reoperation rate due to the vascular ring was $96.9 \%$. Only one case was unable to extubate, hence required tracheostomy.

Surgical treatment provides an uneventful survival in most cases. Backer et al. ${ }^{(16)}$ determined that $92 \%$ of the cases showed an asymptomatic progress in their late follow-up. Furthermore, when Kommerell diverticulum excluded, they have reported only 8 cases that required re-intervention in overall 350 cases. Similarly, Herrin et al. ${ }^{(22)}$ reported necessity of re-invervention in 8 of total 200 cases (115 VATS and 85 thoracoscopy). Seven of these had aortopexy and only two of them were operated via thoracosopy previously.

In our series, we performed aortopexy concurrently and we are in opinion that by doing so, we observed less residual late term respiratory complications. In presence of significant tracheal compression for a long time, there may be irreversible damage to the tracheal structure as well as a localized area of tracheomalacia and cause permanent complaint. Approximately, $70-75 \%$ of symptoms disappear in early period following surgery. ${ }^{(22-23)}$ Bertolini et al. ${ }^{(23)}$ evaluated that the remaining symptoms, which are approximately $\% 30$, tend to disappear at a later period. We also had no residual symptoms except for one case suffering from asthma. However, in this patient there was no significant pressure measured on trachea.

The current study contains limitations of being a retrospective review and designed without concurrent 
control group. In addition, the limited number of patients and lack of data concerning some types of vascular ring classification seem to constitute a separate limitation.

\section{Conclusion}

Vascular ring may manifest in various symptoms and different ages, thus requires awareness for diagnosis. Once the patient was diagnosed surgical treatment is es- sential to prevent irreversible harmful effects. A profound examination and assessment of the anatomy are beneficial regarding the decision of how the surgery should be performed. The goal of the surgery is to relieve the compression over tracheal and/or esophagus by dividing the dislocated vessel while providing sufficient blood flow towards the head. The outcomes after the surgery are satisfactory and lead to uneventful long-term survival.

\section{References}

1. Backer CL, Mavroudis C. Congenital heart surgery nomenclature and data-base project: vascular ring, tracheal stenosis, pectus excavatum. Ann.Thorac. Surg.2000;69:308-18

2. Woods RK, Sharp RJ, Holcomb GW $3^{\text {rd }}$, et al. Vascular anomalies and tracheoesophageal compression: a single institution's 25-year experience. Ann Thorac Surg 2001;72:434-39.

3. Juraszek AL, Guleserian KJ. Common aortic arch anomalies: diagnosis and management. Curr Treat Options Cardiovasc Med 2006;8:414-8.

4. Tola, H., Ozturk, E., Yildiz, O., Erek, E., Haydin, S., Turkvatan, A., et al. (2017). Assessment of children with vascular ring. Pediatrics International, 2017;59:134-40

5. Weiss, A., \& Taragin, B. Double Aortic Arch. Cardiac Imaging, 2014;38.

6. Chun K, Colombani PM, Dudgeon DL, Haller JA Diagnosis and management of congenital vascular rings: a 22-year experience. Ann Thorac Surg 1992;53:597-603

7. Brown JW. Follow-up of surgical correction of aortic arch anomalies causing tracheoesophageal compression: a 38-year single institution experience. J Pediatr Surg 2009;44:1328-32.

8. Backer CL, Mavroudis C. Congenital heart surgery nomenclature and data-base project: Vascular ring, tracheal stenosis, pectus excavatum. Ann. Thorac. Surg. 2000; 69 (4 Suppl): S308-18.
9. Kınoglu B, Sarıglu T, Saltık L et al. [Vascular ring: Results of the surgical management.] Turk Gogus Kalp Damar 1997;5:44-51.

10. Backer C, Mavroudis C, Rigsby CK, Holinger L. Trends in vascular ring surgery. J. Thorac. Cardiovasc. Surg. 2005;129:1339-47.

11. Sturm, Jacquelyn, and Alan P. Knutsen. "Vascular ring presenting as asthma in an 8-year-old." Annals of Allergy, Asthma \& Immunology 108.5 2012: 291-293.

12. Weinberg PM (2001) Aortic arch anomalies. In: Allen HD, Clark EB, Gutgesell HP, Driscoll DJ (eds) Moss and Adams' heart disease in Infants, children, and adolescents 2001. Lippincott Williams \& Wilkins, Philadelphia, pp 707-735

13. Kir, M., Saylam, G. S., Karadas, U., Yilmaz, N., Uzuner, N., et al.(2012) Vascular rings: presentation, imaging strategies, treatment, and outcome. Pediatric cardiology, 2012;33:607-617.

14. Baue AE, Geha AS, Hammond GL, Laks H, Naunheim KS: Gleen's thoracic and cardiovascular surgery. London, Appleton\&Lange 1991; p.963-70.

15. Kinoğlu B., Sarioğlu T., Saltik L., Özkara A., Sarioğlu A., Bilal M. S., et al. Vasküler ring: Cerrahi tedavi ve sonuçlar. Türk Göğüs Kalp Damar Cerrahisi Dergisi, 1997;5: 044-051.

16. Backer, C. L., Mongé, M. C., Popescu, A. R., Eltayeb, O. M., Rigsby, C. K. Vascular rings. In Seminars in pediatric surgery 2016;25:165-75. 
17. Luciano, D., Mitchell, J., Fraisse, A., Lepidi, H.., Ovaert, C. (2015). Kommerell diverticulum should be removed in children with vascular ring and aberrant left subclavian artery. The Annals of thoracic surgery, 2015;100:2293-97.

18. Riggle, Kevin M., Samuel E. Rice-Townsend, John HT Waldhausen. "Thoracoscopic division of vascular rings." Journal of pediatric surgery 2017;52:1113-16.

19. Muller C. O., Ali, L., Matta, R., Montalva, L., Michelet, D., Soudee, S., Bonnard, A. Thoracoscopy Versus Open Surgery for Persistent Ductus Arteriosus and Vascular Ring Anomaly in Neonates and Infants. Journal of Laparoendoscopic \& Advanced Surgical Techniques. (2018)

20. Boehmer J, Berggren H, SunnegardhJ. Results after surgery for vascular rings; a study of 40 consecutive cases operated 1994-2012 in a single institution. Cardiology in the Young 2014;24: pp 163-65. 16-18

21. Suh YJ, Kim GB, Kwon BS, Bae EJ, Noh CI, Lim HG et al. Clinical course of vascular rings and risk factors associated with mortality. Korean Circ J. 2012;42:252-8.

22. Herrin, M. A., Zurakowski, D., Fynn-Thompson, F., Baird, C. W., Pedro, J., Emani, S. M. Outcomes following thoracotomy or thoracoscopic vascular ring division in children and young adults. The Journal of thoracic and cardiovascular surgery, 2017;154: 607-615.

23. Bertolini A, Pelizza A, Panizzon G, Moretti R, Bava CL, Calza G, et al. Vascular rings and slings. Diagnosis and surgical treatment of 49 patients. J Cardiovasc Surg 1987;28:301-12.

Received: $30 / 07 / 2018$

Accepted: $31 / 08 / 2018$

Published: 15/09/2018

Disclosure and conflicts of interest:

The authors declare no conflict of interest.

\section{Funding}

The authors received no financial support for the research and/or authorship of this article.

\section{Corresponding author:}

Çağatay Bilen,

Mail: cagataybilen86@gmail.com 